\title{
Presencia de Receptores de Estrógenos y de Progesterona en el Endometrio de Ovejas Prepúberes. Estudio Inmunocitoquímico
}

\author{
Presence of Estrogen and Progesterone Receptors in Endometrium of Prepubertal Sheeps. \\ Immunocitochemistry Study
}

Adriana Vasconcellos, C.; Néstor Sepúlveda, B.; Jonathan Castillo, J. \& Carlos Rosas, C.

VASCONCEllos, C. A.; SEPÚlVEDA, B. N.; CASTILlO, J. J. \& ROSAS, C. C. Presencia de receptores de estrógeno y de progesterona en el endometrio de ovejas prepúberes. Estudio inmunocitoquímico. Int. J. Morphol., 23(4):393-396, 2005.

RESUMEN: Los receptores esteroidales endometriales tienen un papel esencial en la fisiología reproductiva, siendo ellos determinantes del estado morfuncional del tracto genital y especialmente del endometrio en el cual se implantará el embrión. Se estudió la presencia de receptores de estrógenos y progesterona en el endometrio de ovejas Rommey Marsh prepúberes $(n=3)$. Se obtuvieron muestras de pared uterina para estudios histológico e inmunocitoquímico. El endometrio de la oveja prepúber muestra histológicamente carúnculas, áreas aglandulares de estroma denso rico en fibroblastos que se convertirán en puntos de inserción placentaria durante la gestación y áreas intercarunculares constituidas por endometrio glandular proliferativo de estroma compacto y vasos poco prominentes. La inmunocitoquímica reveló, para los receptores de estrógenos, inmumnoreactividad positiva moderada en el estroma endometrial y muy aisladamente en el epitelio glandular, no encontrándose diferencias entre zona caruncular e intercaruncular. Los receptores de progesterona presentaron positividad estromal siendo algo más intensa en la zona intercaruncular. Concluimos que la oveja Rommey Marsh presenta receptores de estrógenos y progesterona fisiológicamente activos en su endometrio ya en etapa prepúber, o sea, antes de comenzar sus ciclos ováricos, los que podrían ser inducidos y regulados por hormonas exógenas, con el fin de aumentar la eficiencia y prevenir disfunciones reproductivas.

PALABRAS CLAVE: Ovejas; Prepúber; Receptores de estrógenos; Receptores de progesterona.

\section{INTRODUCCIÓN}

Las hormonas esteroidales sexuales actúan a través de sus receptores intracelulares específicos, se unen a ellos e inician la respuesta biológica permitiendo el desarrollo del tracto genital y determinando su estado morfofuncional. Los receptores son regulados por las hormonas (Spencer \& Bazer, 1995; Kimmins \& Mac Laren, 2001 y Robinson et al., 2001) y presentan un complejo mecanismo de control, en el cual es necesario tener varios factores en cuenta, entre ellos la etapa del desarrollo y estadio reproductivo del animal y las diferencias entre las diversas especies.

Garfolo \& Tasende (1996) y Meikle et al., 1997 sugieren la presencia de receptores de estrógenos (RE) y receptores de progesterona (RP) en el endometrio de ovejas prepúberes (Meikle et al., 1997, 2000, 2001). En estudios en otras especies como el perro, los receptores, principal- mente los de progesterona, son muy bajos o indetectables en este estadio del desarrollo (Lessey et al., 1981). Según Meiklel et al. (2004) los rumiantes difieren de otros mamíferos en la distribución de receptores sexuales esteroidales a lo largo del tracto reproductivo.

Ing \& Ott (1999) han observado en el tracto reproductivo de la oveja, la presencia de receptores de estrógeno alfa y beta y se cree que el primero es el receptor proteico que media la clásica acción estrogénica.

Las hormonas sexuales, tanto naturales como sintéticas, actúan sobre los receptores generando modificaciones, dado que su uso farmacológico produciría cambios en ellos es importante determinar su estado previo (Dall Aglio, 1999 y Matamoros et al., 2002). 
El objetivo de nuestro estudio fue evaluar mediante inmunocitoquímica la presencia y distribución de receptores de estrógenos y progesterona en endometrio de ovejas Rommey Marsh prepúberes.

\section{MATERIAL Y MÉTODO}

Se realizó el estudio en ovejas Rommey Marsh. Inmediatamente después del sacrificio, se tomaron muestras de pared uterina de ovejas prepúberes $(n=3)$. Las muestras fueron divididas en dos, una parte fue fijada en Bouin acuoso, para tinciones convencionales y la otra en Methacarn para inmunocitoquimica (ICQ). Las inclusiones se hicieron en paraplast, de las cuales se obtuvieron cortes seriados de 5-7 micras. Los análisis morfológicos se hicieron con hematoxilina y eosina.

La ICQ se hizo con el método del PAP, descrito por Sternberger (1979), según técnica del segundo anticuerpo (AC). En la serie para los receptores estrogénicos se utilizó como $1^{\circ}$ Ac o específico, un monoclonal (Dako, M7047); como $2^{\circ}$ Ac: (-)IgG ratón obtenido en conejo (Dako, Z0109) y el PAP fue PAP-ratón (Dako, P0850). En la serie para los receptores de progesterona se utilizó como $1^{\circ} \mathrm{Ac}$, un policlonal (Dako, A0098), como $2^{\circ}$ Ac: (-) IgG conejo obtenido en cerdo (Dako, Z0196) y PAP fue PAP-conejo (Dako, Z0113). El revelado se hizo con 3,3'- diaminobenzidine (Dako, S-3000 ó Sigma) y $\mathrm{H}_{2} \mathrm{O}_{2}$ por 1-5 minutos, para luego ser deshidratados y montados en Entellan (Merck). Una vez finalizada la reacción, los preparados fueron teñidos con hematoxilina, por 10-30 segundos, como tinción de contraste.

Según la intensidad de la tinción se usó la clasificación de positividad fuerte o marcada (+++), moderada (++), débil o leve (+) y negativa (-). La intensidad de reacción de las muestras fueron comparadas con controles negativos.

El estudio morfológico y las fotografías se realizaron con un microscopio Carl Zeiss, Axiolab,c/cámara MC80 DX.

\section{RESULTADOS}

El endometrio de la oveja prepúber muestra histológicamente carúnculas y áreas intercarunculares. Las carúnculas son zonas aglandulares con estroma denso rico en fibroblastos y las zonas intercarunculares (Fig. 1.) corresponde endometrio proliferativo de estroma compacto con vasos poco prominentes. La inmunocitoquímica reveló para los receptores de estrógenos, inmumnoreactividad positiva moderada en el estroma endometrial y muy aisladamente en el epitelio glandular, no encontrándose diferencias entre zona caruncular e intercaruncular (Figs. 2 y 3). Los RP presentaron positividad estromal siendo algo más intensa en la zona intercaruncular. (Fig. 4).

\section{DISCUSIÓN}

Conocida es la importancia de los receptores esteroidales en los mecanismos reproductivos. El endometrio es muy sensible a la acción de las hormonas sexuales, las que modifican con rapidez y versatilidad sus características estructurales. La importante función de sus receptores en la dinámica de los cambios son determinantes del estado morfuncional del tracto genital, dependiendo la respuesta celular de distintos factores que afectan la sensibilidad y función de los tejidos, como son el número y afinidad a sus receptores (tipos y subtipos de ellos), el estado hormonal, la expresión selectiva en los órganos diana, la etapa del desarrollo y el estadio reproductivo en que se encuentre el animal y la diferencia entre las diversas especies.

La presencia de RE y RP ha sido estudiada en el tracto genital de distintas especies y se han demostrado diferencias entre ellas. Mientras que en las ovejas se encuentran altas concentraciones de RE y RP en el útero y oviducto de animales prepúberes (Garfolo \& Tasende y Meikle et al., 1997, 2000, 2001, 2004) en perros (Lessey et al.) los RP son en este estadio del desarrollo bajos o indetectables tanto en el útero como en el oviducto. En primates y roedores la expresión de los receptores en el oviducto es dependiente de las hormonas (Slayden et al., 1993) y su regulación sería en el oviducto similar a la del útero, aumentaría con los estrógenos y disminuiría con la progesterona.

Meikle et al. (2004) consideran que los esteroides gonadales regulan a sus RE y RP de forma diferente a lo largo del tracto genital de la oveja, sugiriendo que las target cells de los tejidos pueden modular distintas respuestas a los mismos niveles sanguíneos circulantes de hormona.

En la práctica veterinaria se ha generalizado el empleo de hormonas sexuales sintéticas para producir inducción del celo y sincronizaciones, como método para mejorar la eficiencia reproductiva (Córdova et al., 1999 y Matamoros et al.), estos fármacos actúan sobre los receptores generando modificaciones en ellos. Es importante, por lo tanto, la evaluación previa de los receptores sobre los que actúan estas hormonas para el diagnóstico de problemas reproductivos en los animales domésticos, incluyendo al bovino y ovino. 


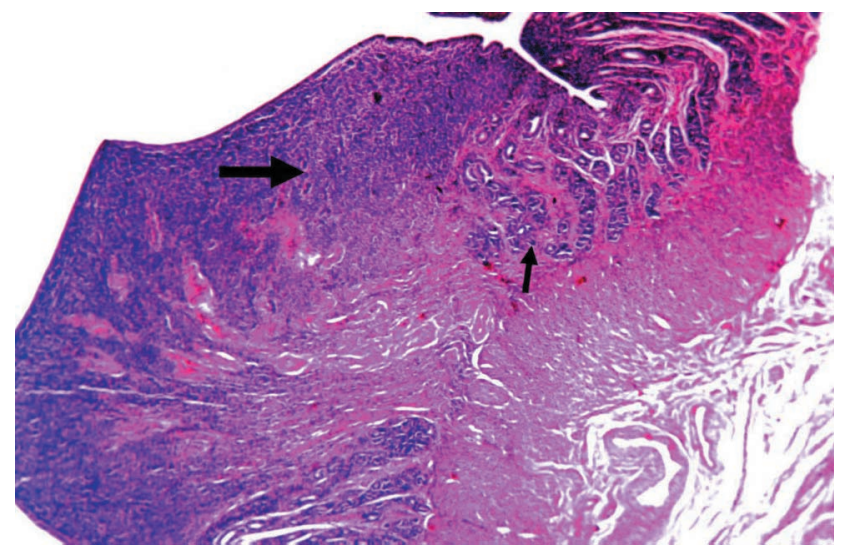

Fig. 1. Endometrio con zonas intercarunculares glandulares (flecha delgada) y áreas carunculares aglandulares (flecha gruesa) de oveja prepúber. H-E 40x.

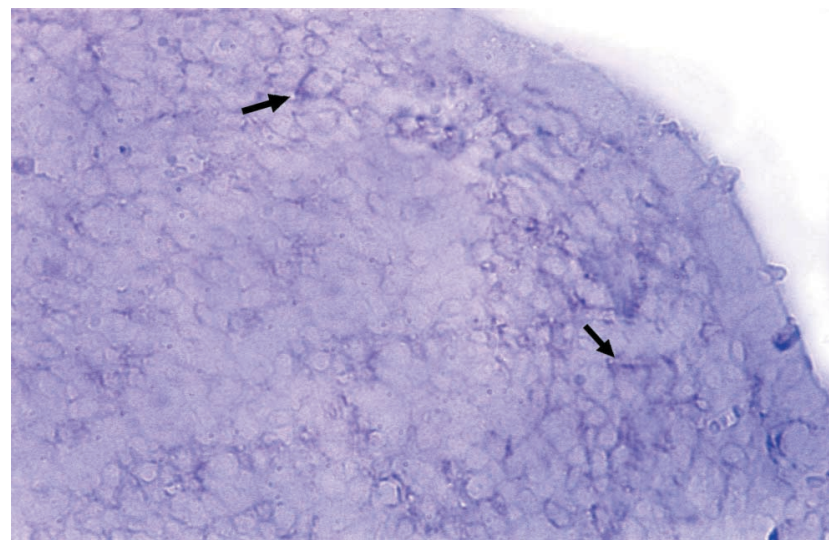

Fig. 3 Localización inmunocitoquímica de receptores de estrógenos en células de estroma endometrial. Se observa inmuno- reactividad leve a moderada (flechas), que indica la presencia de sus receptores. 400x.

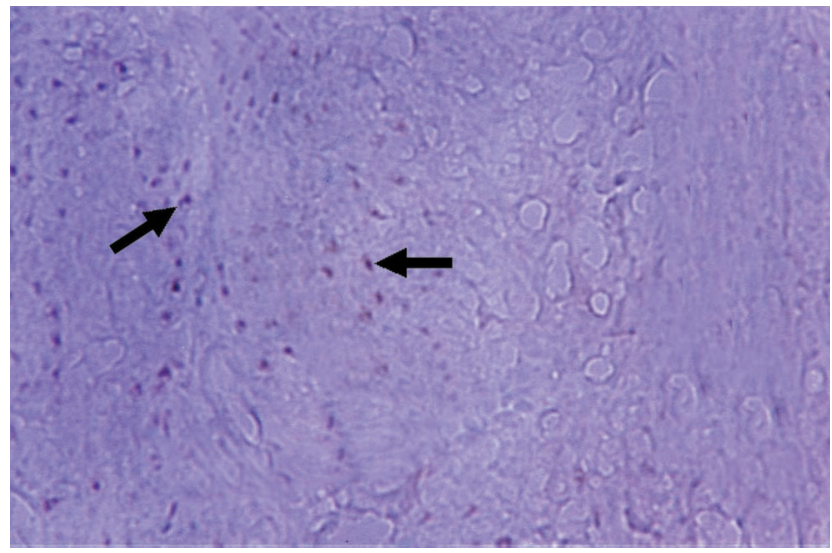

Fig. 5. Localización inmunocitoquímica de receptores de progesterona en células de estroma endometrial. Se observa inmuno- reactividad leve a moderada (flechas), que indica la presencia de sus receptores. 400x.

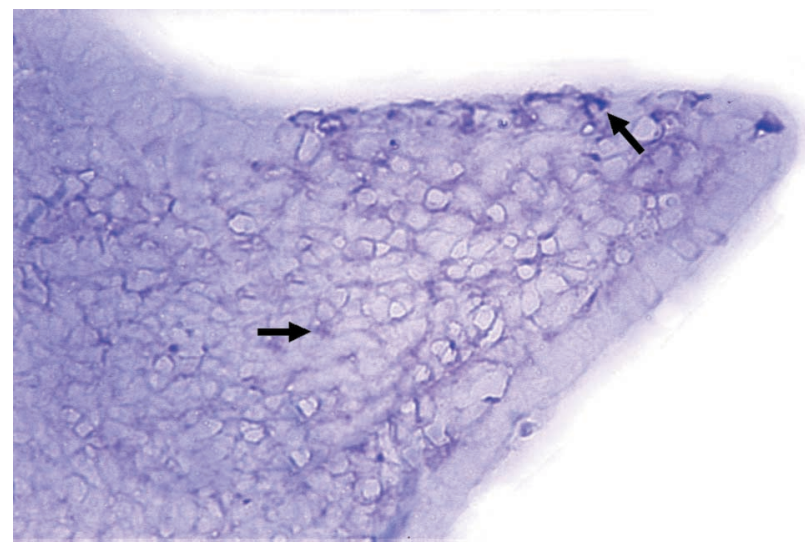

Fig. 2 Localización inmunocitoquímica de receptores de estrógenos en células de estroma endometrial. Se observas inmunoreactividad leve a moderada (flechas), que indica la presencia de sus receptores. $400 x$.

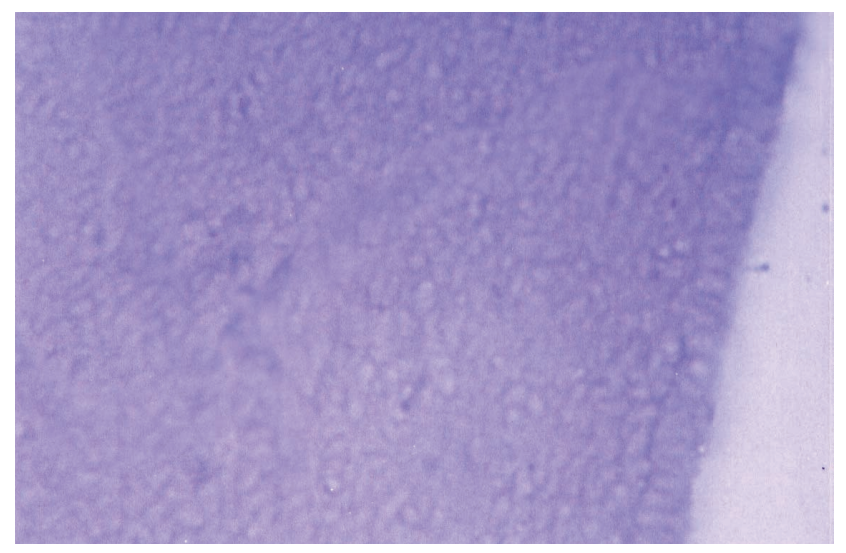

Fig. 4. Control negativo de estrógenos.

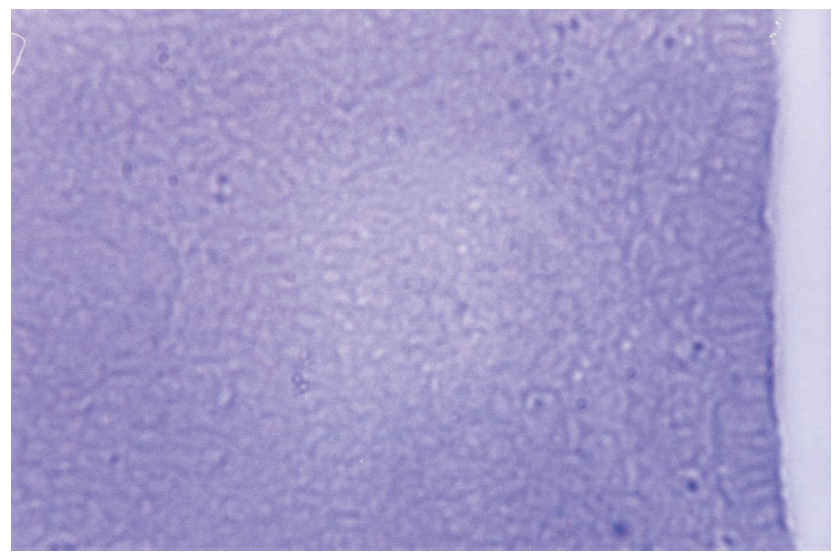

Fig. 6. Control negativo de progesterona. 
Por los resultados observados en este estudio concluimos que la presencia de receptores de estrógenos y progesterona en endometrio de ovejas prepuberes es siem- pre detectable, lo que lo hace susceptible a la acción de hormonas exógenas mejorando así la eficiencia reproductiva y previniendo disfunciones.

VASCONCELLOS, C.A.; SEPÚLVEDA, B. N.; CASTILLO, J.J. \& ROSAS, C. C. Presence of estrogen and progesterone receptors in endometrium of prepubertal sheep. Inmunocitochemestry study. Int. J. Morphol., 23(4):393-396, 2005.

SUMMARY: The endometrial esteroid receptors play an essential paper in the reproductive physiology being them decisive on the morophuncional state of the genital tract and especially of the endometrium in which the embryo will be implanted. We studied the presence of estrogens and progesterone receptors in the endometrium of Rommey Marsh prepubertal sheep $(n=3)$ samples of uterine wall were obtained for histologycal and and Inmunocitochemestry study. The endometrium of the prepubertal sheep shows histologycaly caruncles, aglandulares areas of dense estroma rich in fibroblastos, that will become points of placental insertation during the gestation and intercarunculares areas constituted by endometrium glandular proliferative of compact estroma and not very prominent vessels. The Inmunocitochemestry revealed for the estrogens receptors inmumnoreactivity positive moderated in the estroma endometrial and very scattered in the glandular epithelium, not being differences between area caruncular and intercaruncular. The progesterone receptors presented positive inmunoreactivity estromal being more intense in the intercaruncular area We conclude that the sheep Rommey Marsh shows estrogens and progesterone receptors physiologically active in its endometrium already in prepubertal stage this is before beginning its ovarian cycles, those could be induced and regulated by exogenous hormones with the purpose of to increase the efficiency and to prevent reproductive disfunctiones.

KEY WORDS: Sheep; Prepubertal; Estrogens receptors; Progesterone receptors.

\section{REFERENCIAS BIBLIOGRÁFICAS}

Dall Aglio, C. Inmunohistochemical localization of estrogen and progesterone receptors in the cow. Anat. Histol. Embryol., 28:3757, 1999.

Córdova Izquierdo, A.; Ruiz Lang, G.; Saltijeral, J.; Pérez Gutierrez, J. F. \& Degefa, T. Inducción y sincronización de celos en ovejas criollas anéstrcas estacionales con esponjas vaginales impregnadas en FGA y PMSG Inyectable. Arch. Zootec., 48:437-40, 1999.

Garfolo, E.G. \& Tasende, C. Uterine estrogen and progesterone receptors in prepuberal ewe distribution in myometrium, endometrium, and caruncles. Vet. Res., 27:177-3, 1996.

Ing, N. H.; \& Ott, T. L. Estradiol up-regulates estrogen receptor- a messenger ribonucleic acid in sheep endometrium by increasing its stability. Biol. Reprod., 60:134-9, 1999.

Kimmins, S. \& Mac Laren, L. A. Oestrous cycly and pregnancy effects on the distribution of oestrogen and progesterone receptors in bovine endometrium. Placenta 22:742-8, 2001.

Lessey, B. A.; Wahawisan, R. \& Gorell, T. A. Hormone regulation of cytoplasmic estrogen and progesterone receptors in the beagle uterus and oviduct. Moll. Cell. Endocrinol., 21(2):171-80, 1981.

Matamoros, R.; Gómez, C. \& Andaur, M. Hormonas de Utilidad Diagnóstica en Medicina Veterinaria. Arch. Med. Vet., 34:527.2002.

Meikle, A.; Tasende, C.; Rodríguez, M. \& Garófalo, E. G. Effects of estradiol and progesterone on the reproductive trac and on uterine sex steroid receptors in female lambs. Theriogenology, 48:110513,1997

Meikle, A.; Forsberg, M.; Shalin, L.; Masironi, B.; Tasende, C.; Rodríguez-Piñon, M. \& Garófalo, E. G. A biphasic action of estradiol on estrogen and progesterone receptor expression in the lamb uterus. Reprod. Nutr. Dev., 40: 283-93, 2000.

Meikle, A.; Garófalo, E. G.; Rodríguez- Piñon, M.; Tasende, C. \& Sahlin, L. Regulation by gonadal steroids of estrogen and progesterone receptors along the reproductive trac in lambs. Acta Vet. Scand., 42:131-9, 2001.

Meikle, A.; Tasende, C.; Sosa, C. \& Garófalo, E. G. The rol of sex steroid receptors in sheep female reproductive physiology. Reprod. Nutr. Dev., 16:385-94, 2004.

Robinson, R. S.; Mann, G. E.; Lamming, G. E. \& Wathes, D. C. Expression of oxytocin, oestrogen and progesterone receptors in uterine biopsy samples through the oestrus cycle and early pregnancy in cows. Reproducction, 122: 965-79, 2001.

Slayden, O. D.; Hirst, J. J. \& Brenner, R. M. Estrogen action in the reproductive tract of rheus monkeysduring during antiprogestin treatment. Endocrinology, 132:1845-56, 1993.

Spencer, T.E. \& Bazer, F.W. Temporal and spatial alterations in uterine estrogen receptor and progesterone receptor gene expression during the estrous cycle and early pregnancy in the ewe. Biol. Reprod., 53:1527-43, 1995.

Sternberger, L. A. The unlabled antibody (PAP) method,introduction. J. Histochem. Cytochem., 27(12):1657-65, 1979.

Dirección para correspondencia:

Prof. Dra. Adriana Vasconcellos Costa

Facultad de Medicina Universidad de La Frontera

Casilla 54-D

Temuco - CHILE

Email:avascon@ufro.cl

Recibido : 30-08-2005

Aceptado: 15-10-2005 